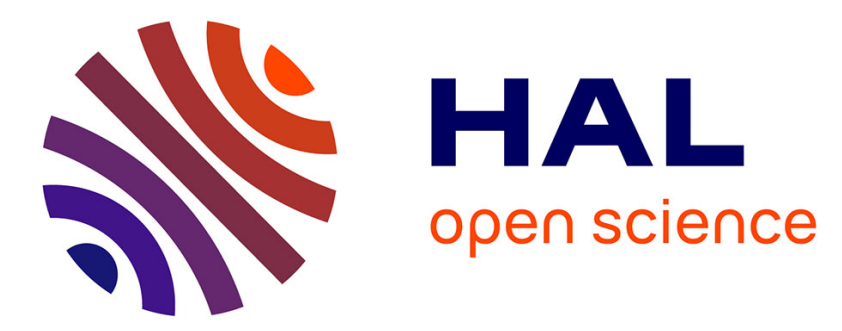

\title{
UN RÈGLEMENT POUR DES JOUETS SÉCURITAIRES POUR LES OREILLES DE NOS ENFANTS
}

T. Leroux, C. Laroche

\section{- To cite this version:}

T. Leroux, C. Laroche. UN RÈGLEMENT POUR DES JOUETS SÉCURITAIRES POUR LES OREILLES DE NOS ENFANTS. Journal de Physique IV Proceedings, 1992, 02 (C1), pp.C1-241-C1244. 10.1051/jp4:1992150 . jpa-00251221

\section{HAL Id: jpa-00251221 https://hal.science/jpa-00251221}

Submitted on 1 Jan 1992

HAL is a multi-disciplinary open access archive for the deposit and dissemination of scientific research documents, whether they are published or not. The documents may come from teaching and research institutions in France or abroad, or from public or private research centers.
L'archive ouverte pluridisciplinaire HAL, est destinée au dépôt et à la diffusion de documents scientifiques de niveau recherche, publiés ou non, émanant des établissements d'enseignement et de recherche français ou étrangers, des laboratoires publics ou privés. 


\title{
UN RÈGLEMENT POUR DES JOUETS SÉCURITAIRES POUR LES OREILLES DE NOS ENFANTS
}

\author{
T. LEROUX et C. LAROCHE \\ Sonométric Inc. Expertise-Conseil en Bruit et Audition, 5757 avenue Decelles, Bureau 514, Montréal \\ (Québec) H3S 2C3, Canada
}

\begin{abstract}
The Canadian Federal Act prohibiting sale of hazardous products limits the sound level emitted by toys, equipment and other products for use by a child in learning or play to $100 \mathrm{~dB}$. The scientific literature shows that this legal criteria is not safe for children regarding the risk of acquiring hearing loss. Based on a safe limit of $75 \mathrm{dBA}$, more than $85 \%$ of the toys available on the Canadian market are not safe and may induce hearing loss and other adverse effects on the long term. There is evidence that this situation is probably the same or even worse in United States and many European countries. Toys emitting noise from explosive sources have been repeatedly demonstrated in case studies to be very harmful to hearing even after one short exposure. These toys are beyond the scope of the Canadian Act. In fact, the current Act authorizes the sale of hazardous noisy toys that may contribute to some extent to deafness in children. Two factors can explain the inefficiency of the Canadian Act: the current limit for sound level is too lax and the regulation does not include a precise and specific method for determination of the sound level produced by a given toy. Based on a review of literature and previous studies done by the Groupe d'Acoustique de l'Université de Montréal, a new regulation and a measurement method have been proposed to Consumers and Corporate Affairs Canada in order to correct those deficiencies. The limit for sound level has been set to $75 \mathrm{dBA}$, a world wide accepted safe limit, regardless of the exposure duration. Concerning the method of measurement, the Canadian Standard Association standard on noise emitted by small appliances [CSA Z107.71-M1981] has been proposed. This paper will present the results of a review of the literature on the topic including the examination of the actual american and european countries legislations. We will also describe a proposal for a new canadian regulation for noisy toys.
\end{abstract}

\section{Introduction}

Depuis quelques années, on s'intéresse aux niveaux de bruit que produisent les jouets sonores utilisés par les enfants et au risque d'atteinte auditive potentiel que pourraient représenter certains d'entre eux. Des études, pilotées par le Groupe d'Acoustique de l'Université de Montréal (GAUM) $(1,2)$, montrent qu'une proportion importante des jouets sonores disponibles sur le marché canadien émettent des bruits dont le niveau peut comporter des risques d'atteinte à l'audition des enfants utilisateurs. Ces études soulèvent, de plus, que la réglementation canadienne actuelle (chapitre $\mathrm{H}-3$ de la Loi fédérale interdisant la vente, l'annonce et l'importation de produits dangereux)(3) est imprécise et permet à une grande proportion de ces jouets de se retrouver, tout-à-fait légalement, entre les mains des enfants. Afin d'éliminer ce risque d'atteinte inutile, nous proposons une nouvelle réglementation ayant comme objectif la protection de la santé des enfants. Cette proposition, qu'étudie actuellement les autorités gouvernementales canadiennes, est basée sur une revue de la littérature scientifique s'intéressant 1) au risque d'atteinte à l'audition chez l'enfant variant en fonction des niveaux de pression sonore produits par les jouets, 2) aux effets extra-auditifs de l'exposition au bruit chez l'enfant et 3 ) à la réglementation canadienne et internationale portant sur les jouets sonores. 


\section{Le risque d'atteinte à l'audition chez l'enfant et l'exposition aux bruits produits par les jouets}

Le risque d'atteinte à l'audition et l'ampleur de la perte auditive varient en fonction des paramètres de l'exposition au bruit: le niveau de pression sonore du bruit et la durée d'exposition. Une augmentation de l'un ou l'autre de ces paramètres augmente le risque d'atteinte et l'ampleur de la perte auditive (4). Ainsi, une exposition de courte durée à un bruit d'amplitude très élevée et une exposition prolongée à un bruit d'amplitude modérée représentent un risque important d'atteinte à l'audition et peuvent causer une perte d'acuité auditive considérable. La nature de l'atteinte auditive est permanente, irréversible et actuellement incurable médicalement.

On admet que le risque d'atteinte à l'audition est négligeable pour un niveau sonore quotidien ne dépassant pas $75 \mathrm{~dB}(\mathrm{~A})$ pour 8 heures d'exposition ou $70 \mathrm{~dB}(\mathrm{~A})$ pour 24 heures (5). Afin de respecter cette limite sécuritaire, il faut limiter la durée d'exposition si le niveau sonore est plus important que $75 \mathrm{~dB}(\mathrm{~A})$. On admet donc $78 \mathrm{~dB}(\mathrm{~A})$ pour 4 heures, $81 \mathrm{~dB}(\mathrm{~A})$ pour 2 heures, 84 pour $\mathrm{dB}(\mathrm{A})$ pour 1 heure, etc. En fait; on additionne $3 \mathrm{~dB}$ pour chaque dédoublement de la durée d'exposition.

Ces limites sécuritaires sont valides pour des bruits de type continus ou quasi-continus et ne s'appliquent pas pour des bruits d'impact ou à caractère impulsionnel. Pour prédire la nocivité de ces derniers types de bruits, il faut tenir compte de plusieurs autres caractéristiques (durée de croissance et de décroissance, spectre, etc.). Selon les résultats des travaux menés par le GAUM $(6,7,8)$, les niveaux qui limiteraient, chez l'adulte, l'acquisition de toute perte auditive, même temporaire, après 2000 coups, seraient les suivants: $95 \mathrm{~dB}(\mathrm{~A})$ pour des bruits d'impact dont le contenu en fréquence est centré à $3 \mathrm{kHz}, 100 \mathrm{~dB}(\mathrm{~A})$ pour des bruits d'impact dont le contenu en fréquence est centré à $1 \mathrm{kHz}, 125 \mathrm{~dB}(\mathrm{~A})$ pour des impulsions dont le contenu en fréquence est centré à $3 \mathrm{kHz}$ et $130 \mathrm{~dB}(\mathrm{~A})$ pour des impulsions dont le contenu en fréquence est centré à $1 \mathrm{kHz}$.

Ces limites sécuritaires sont basées sur des données obtenues auprès d'adultes oeuvrant en milieu industriel bruyant. II semble que celles-ci ne puissent être appliquées directement aux enfants en raison des différences que nous impose leur système auditif. Contrairement à celui de l'adulte, le système auditif de l'enfant présente des caractéristiques dynamiques de développement qui, à certains égards, augmentent le risque d'atteinte $(9,10,11,12)$. Ces recherches mettent en évidence que les limites sécuritaires d'exposition admises pour l'adulte ne tiennent pas compte d'une éventuelle période de susceptibilité accrue au trauma acoustique chez l'enfant.

Tous les articles répertoriés à travers le monde et portant sur le niveau sonore des jouets montrent, à chaque fois, la présence de jouets potentiellement dangereux pour l'audition selon d'une part, l'opinion des auteurs et, d'autre part, les limites sécuritaires énoncées auparavant $(1,13,14,15,16,17,18,19,20,21)$. L'ensemble de ces données montrent clairement que les jouets sonores tels les pièces pyrotechniques à main et les jouets imitant les armes à feu représentent un danger potentiel pour l'audition des enfants utilisateurs. Des études de cas nous montrent même que ce risque se matérialise pour une proportion non-négligeable d'enfants après une seule exposition $(22,23)$. Par ailleurs, une grande variété de jouets, à première vue inoffensifs, allant du simple hochet de bébé à l'imitation du camion de pompier peuvent produire des niveaux sonores présentant un risque réel d'atteinte à l'audition.

\section{Les effets extra-auditifs de l'exposition au bruit chez l'enfant}

En plus des effets nocifs sur la santé auditive, la présence du bruit dans l'environnement de l'enfant peut engendrer d'autres types de problèmes moins spécifiques et donc plus difficiles à identifier. L'interférence à la communication causée par le bruit a été identifiée comme une des causes du ralentissement de l'acquisition des capacités de traitement des éléments de la parole, de l'attention sélective et des stratégies d'écoute (24). D'autres études ont repris ces éléments pour étudier le développement d'habiletés cognitives comme la lecture et l'écriture. L'exposition au bruit entraînerait des difficultés de concentration menaçant ces apprentissages $(25,26)$. La présence de 
niveaux de bruit élevés dans les aires de jeux et d'apprentissage peut contribuer à l'apparition plus fréquente de troubles de l'apprentissage.

De plus, le niveau de pression acoustique où apparaissent ces effets extra-auditifs est modéré si l'on considère, par exemple, qu'au-delà d'un niveau de bruit ambiant de $45 \mathrm{~dB}(\mathrm{~A})$ à l'intérieur d'une pièce, lintelligibilité de la parole commence à se dégrader et chute abruptement autour de $70 \mathrm{~dB}(\mathrm{~A})$ (27). Ces niveaux sont éloignés des limites sécuritaires proposées pour éviter tout risque d'atteinte à l'audition. Toutefois, parce que les enfants sont en pleine période de développement des fonctions du langage, il nous apparaît très important de prendre en compte, non seulement la notion de risque, mais également la qualité de l'environnement sonore. A cet égard, les jouets sonores présentant des niveaux de bruit élevés contribuent à la dégradation de l'environnement de l'enfant.

\section{La réglementation canadienne et internationale portant sur les jouets sonores}

Le tableau I fait la synthèse des dispositions réglementaires portant sur les jouets sonores à travers les monde. Un nombre restreint de pays possède une législation spécifique aux niveaux sonores produits par les jouets. Dans la majoritè des cas, les règlements s'avèrent imprécis dans leur application ou, comme dans le cas de la loi canadienne, utilise une limite réglementaire n'ayant aucun fondement scientifique valable.

Tableau I - Les règlements portant sur les jouets sonores à travers le monde

\begin{tabular}{|l|l|l|}
\hline Pays & Niveau & Portée législative \\
\hline Canada(3) & $100 \mathrm{~dB}$ & Jouets explosifs exclus \\
\hline Norvège/Suède(28) & $\begin{array}{l}90 \mathrm{dBA} \text { à } 50 \mathrm{~cm} \\
135 \mathrm{~dB} \text { acrête }\end{array}$ & Tous les jouets \\
\hline Pays-Bas(29) & $\begin{array}{c}\text { Jugement d'un } \\
\text { inspecteur }\end{array}$ & Tous les jouets \\
\hline Etats-Unis(30) & $138 \mathrm{~dB}$ crête & $\begin{array}{l}\text { Amorces explosives } \\
\text { seulement }\end{array}$ \\
\hline
\end{tabular}

\section{La proposition de règlement canadien}

Notre proposition de réglementation s'appuie d'abord sur le principe que les jouets et les jeux ne devraient entraîner aucun risque pour la santé auditive, la santé en général ni entraîner aucune perturbation indésirable du développement de l'enfant. Considérant la fonction éducative et ludique de ces objets, il n'existe aucune raison valable pour justifier le fait que des jouets émettent un niveau de bruit potentiellement dangereux pour la santé. Certains jouets produisant des bruits impulsionnels de nature explosives présentent un très grand risque d'atteinte à l'audition même pour une seule exposition. Les limites sécuritaires d'exposition au bruit admises internationalement pour l'adulte pourraient ne pas être tout-à-fait adaptées à la population des enfants. Dans l'attente des précisions scientifiques nécessaires, ces limites devraient constituer un seuil à ne jamais dépasser. II nous apparaît illusoire de vouloir limiter le temps d'utilisation des jouets sonores. A cet égard, la limite réglementaire devrait s'appliquer peu importe le temps d'utilisation prévu par le manufacturier du jouet. La réglementation doit s'appliquer à tous les jouets sans exception. 
La législation devrait interdire qu'un jouet émette un bruit continu ou quasi-continu ou une combinaison de bruits dont le niveau dépasse $75 \mathrm{~dB}(\mathrm{~A})$ pour l'une ou l'autre de ces distances: à l'oreille, à $10 \mathrm{~cm}, \grave{a} 30 \mathrm{~cm}$ ou à $1 \mathrm{~m}$. Les jouets produisant des impacts ou des impulsions ne devraient émettre aucun bruit de ce type à des niveaux supérieurs à $95 \mathrm{~dB}$ crête à l'oreille, à $10 \mathrm{~cm}$, à $30 \mathrm{~cm}$ ou à $1 \mathrm{~m}$. La distance est choisie en fonction de l'utilisation habituelle du jouet. Les jouets émettant des impulsions de nature explosive devraient être bannis.

Un code d'essai des jouets uniforme et précis doit être inclus dans le texte légal pour assurer une évaluation valide et fiable des niveaux de pression sonore pour tous les manufacturiers. A cet effet, nous proposons l'utilisation de la norme canadienne CSA 2107.71-M1981 Measurement and Rating of the Noise Output of Consumer SMall Appliances . Cette norme nous est apparue, après essai en laboratoire, uniformément applicable à tous les types de jouets. En utilisant la puissance acoustique pour la mesure des bruits à caractère continu ou quasi-continu, elle permet de calculer a posteriori le niveau de pression sonore dans un local typique à n'importe quelle distance de la source. Pour les bruits impulsionnels, la mesure du niveau crête doit être utilisée.

\section{Conclusion}

La présente proposition de réglementation vise l'élimination des jouets bruyants pouvant constituer un danger pour la santé des utilisateurs. Son application pourrait également contribuer à améliorer la qualitè de l'environnement sonore de nos enfants. Nous souhaitons que ces travaux incitent d'autres pays industrialisés à s'engager dans la mème voie.

Projet commandé par l'Association des Consommateurs du Quebec et financé par Consommations et Corporation Canada.

[1] Lacombe, F. Universitè de Montréal (1989).

[2] Brochu, S., Lacombe, F. et Poisson, M. Université de Montréal (1983).

[3] La Gazette du Canada II. 1968-69. c.42, annexe I (1980) .

[4] ISO 1999 Organisation Internationale de Normalisation (1985).

[5] Öganisation Mondiale de la Santé (1980).

[6] Laroche, C. et Hétu, R. Acustica 70 (1990).

[7] Laroche, C. et Hétu, R. Premier Congrès Français d'Acoustique (1990).

[8] Laroche, C., Hétu, R. et Poirier, S. J. Acoust. Soc. Am. 85 (1989).

[9] Douek, E., Dodson, H.C., Bannister, L.H. et Ashcroft, P. The Lancet 20 (1976).

[10] Stennert, E., Schulte, F.J. et Vollrath, M. Early Human Development (1977).

[11] Bess, F. H., Peek, B.F. et Chapman, B. Pediatrics 63 (1979).

[12] Uziel, A. Acta Otolaryngol (Stockholm)s.421 (1985).

[13] Ward, W.D. et Glorig, A. Laryngoscope 71 (1961).

[14] Gjaevenes, K. J. Acoust. Soc. Am. 39 (1966).

[15] Hodge, D.C. et McCommons, R.B. J. Acoust. Soc. Am. 40 (1966).

[16] Bess, F.H., Powell, R.L. Clin. Pediatr.11 (1972).

[17] Marshall, L. et Brandt, J.F. J. Speech and Hearing Disorders 34 (1973).

[18] Gjaevenes, K., Moseng, J. et Noedahl, T. Scand. Audiol. 3 (1974).

[19] Axelsson, A. et Jerson, T. The National Swedish Board for Consumer Policies (1983).

[20] Plakke, B.L. 4 (1983).

[21] Axelsson, A., Jerson, T. Pediatrics 76 (1985).

[22] Gupta, D. et Vishwakarma, S.K. Laryngoscope 99 (1989).

[23] Cummings, R.J. The Hearing Journal 43 (1990).

[24] Mills, J.H. J. Acoust. Soc. Am. 58 (1975).

[25] Deutsch, C.P. Merrill-Palmer Quarterly of Behavior and Development 10 (1964).

[26] Cohen, S., Glass, D.C. et Singer, J.E. J. of Experimental Social Psychology 9 (1973).

[27] Environmental Protection Agency (US) (1974).

[28] Axelsson, A. Personnal communication (1990)

[29] Commodities Act, Netherland.

[30] F 963-86. American Society for Testing and Materials (1986). 